\title{
Erratum to: Fertilizer potential of thin stillage from wheat-based ethanol production
}

\author{
Khaled D. Alotaibi $•$ Jeff J. Schoenau $\cdot$ Xiying Hao
}

Published online: 15 March 2015

(C) Springer Science+Business Media New York 2015

Erratum to: Bioenergy Res (2014) 7: 1421-1429

DOI 10.1007/s12155-014-9473-1

Unfortunately, the original version of this article contained a mistake in Fig. 3. The correct version is provided below.

The online version of the original article can be found at http://dx.doi.org/ 10.1007/s12155-014-9473-1.

K. D. Alotaibi · J. J. Schoenau Department of Soil Science, University of Saskatchewan, 51 Campus Drive, Saskatoon, SK S7N 5A8, Canada

K. D. Alotaibi $(\square)$

Department of Soil Science, King Saud University, Box 2460,

Riyadh 11451, Saudi Arabia

e-mail: kalotaib@gmail.com

X. Hao

Agriculture and Agri-Food Canada, Lethbridge Research Centre, Environmental Health, 5403-1st Ave, South, Lethbridge,

AB T1J4B1, Canada

Present Address:

K. D. Alotaibi

Department of Soil Science, University of Saskatchewan, 51 Campus

Drive, Saskatoon, SK, Canada S7N 5A8 


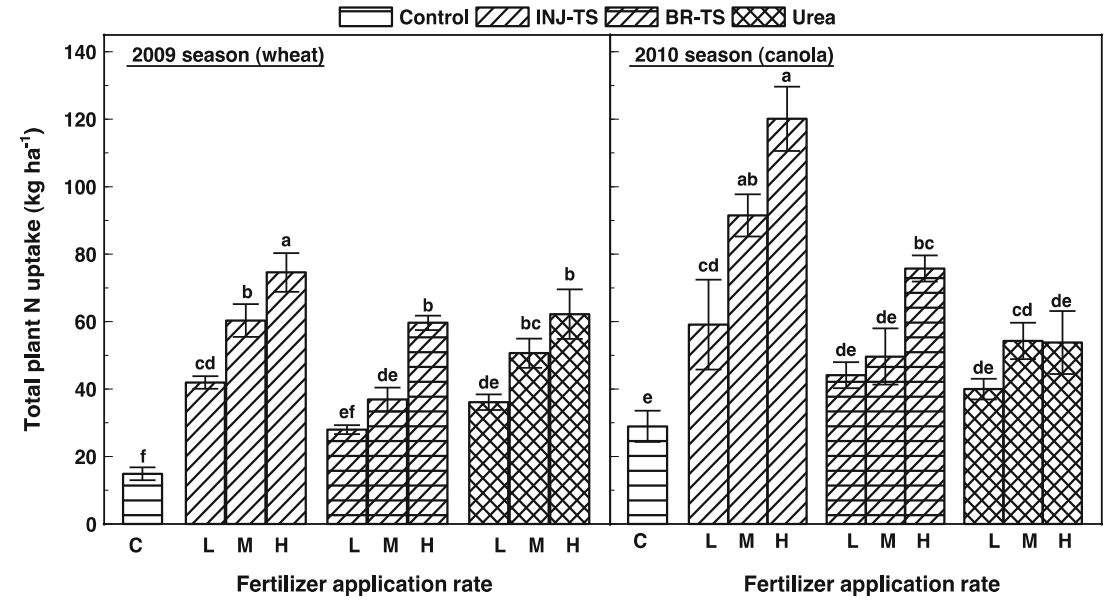

Fig. 3 Total plant $\mathrm{N}$ uptake responses to experimental treatments during 2009 and 2010 growing seasons at Dixon, SK. The experimental rates: low $(L)$, medium $(M)$ and high $(H)$ in addition to a control. For a season, bars sharing the same letter among treatments are not significantly different according to LSD test $(P \leq 0.05)$. Errors bars represent standard treatments included injected thin stillage (INJ-TS), broadcast and error of the mean $(n=4)$ 